\title{
The origin and pulsations of extreme helium stars $\dagger$
}

\author{
C. Simon Jeffery
}

Armagh Observatory, College Hill, Armagh BT61 9DG, Northern Ireland, UK

\begin{abstract}
Stars consume hydrogen in their interiors but, generally speaking, their surfaces continue to contain some $70 \%$ hydrogen (by mass) throughout their lives. Nevertheless, many types of star can be found with hydrogen-deficient surfaces, in some cases with as little as one hydrogen atom in 10000 . Amongst these, the luminous B- and A-type extreme helium stars are genuinely rare; only $\sim 15$ are known within a very substantial volume of the Galaxy.

Evidence from surface composition suggests a connection to the cooler $\mathrm{R} \mathrm{CrB}$ variables and some of the hotter helium-rich subdwarf $\mathrm{O}$ stars. Arguments currently favour an origin in the merger of two white dwarfs; thus there are also connections with AM CVn variables and Type Ia supernovae. Pulsations in many extreme helium stars provide an opportune window into their interiors. These pulsations have unusual properties, some being "strange" modes, and others being driven by $Z$-bump opacities. They have the potential to deliver distance-independent masses and to provide a unique view of pulsation physics.

We review the evolutionary origin and pulsations of these stars, and introduce recent progress and continuing challenges.
\end{abstract}

Keywords. stars: early-type, stars: chemically-peculiar, stars: supergiants, stars: white dwarfs, stars: evolution, stars: oscillations, stars: variable: other, stars: individual: V652 Her, FQ Aqr

\section{Extreme helium stars}

Observing from McDonald Observatory at a maximum altitude of $14^{\circ}$, Popper (1942) reported the B2 star HD 124448 to "show no hydrogen lines, either in absorption or in emission, although the helium lines are sharp and strong ... The abundance of hydrogen appears to be very low in the atmosphere of this star". Indeed, HD 124448 turned out to be the first of around 20 B- and early A- supergiants, with apparent magnitude $9.3<V<12.6$, in which hydrogen comprises less than 1 part per thousand of the atmosphere. In terms of kinematics, metallicity and galactic distribution, they have the properties of the Galactic bulge (Jeffery et al. 1987). With luminosities approximately ten thousand times solar, the number count is complete for the observable parts of the Galaxy (fainter stars would lie beyond). In addition to their low hydrogen abundances, the true "extreme helium stars" (EHes) show atmospheres which are enriched in nitrogen by a factor ten, carbon by between one and three parts per hundred, and sometimes oxygen by a similar amount. The combination of extreme surface composition and extreme rarity makes these stars interesting, and presents a challenge for the theory of stellar evolution.

The general properties of EHes have prompted suggestions of a connection to the cooler R Coronae Borealis stars (Schönberner 1975), which are better known for their spectacular and unpredictable light variability (Pigott \& Englefield 1797). Figure 1 shows the distribution by surface gravity and effective temperature of several classes of hydrogen-

$\dagger$ The full version of this paper, including additional sections on the asteroarchæology and origin of EHes, is available online at ArXiv: 1311.1635. 


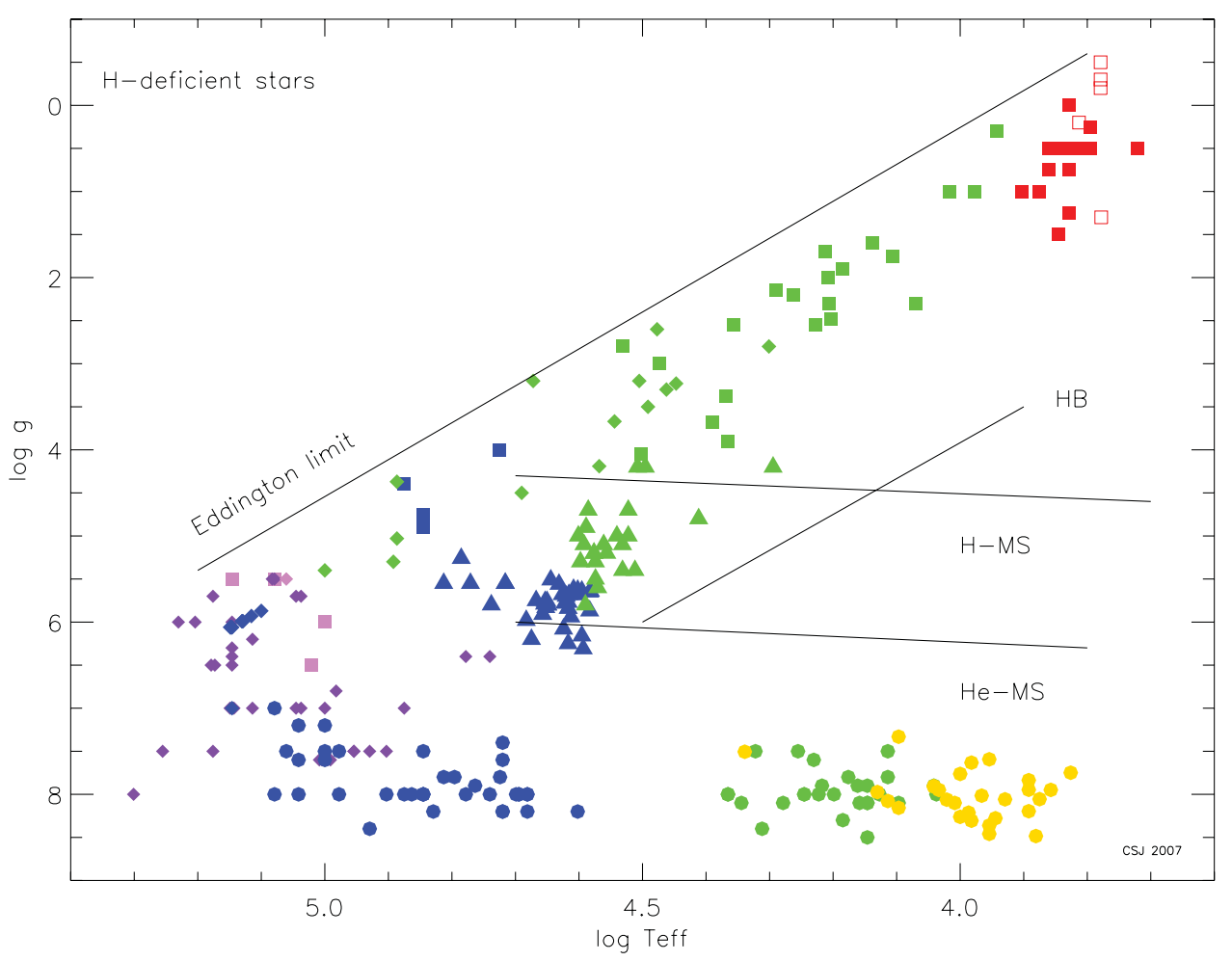

Figure 1. The $\log g-\log T_{\text {eff }}$ diagram for several classes of low-mass hydrogen-deficient stars described in detail by Jeffery (2008a). EHe stars are denoted by squares (light grey, green online), as are RCB (dark grey, red online), $\mathrm{HdC}$ (open squares), $\mathrm{HesdO}^{+}$(back, blue online) and $\mathrm{O}(\mathrm{He})$ (mid-grey, violet online) stars.

deficient star; stars falling on an imaginary line parallel to the Eddington limit would have the same luminosity-to-mass ratio, corresponding to the evolutionary path of a giant contracting to become a white dwarf. Links between non-variable hydrogen-deficient carbon (HdC), R Coronae Borealis (RCB), extreme helium (EHe), luminous helium-rich subdwarf $\mathrm{O}\left(\mathrm{HesdO}^{+}\right)$and $\mathrm{O}(\mathrm{He})$ stars have all been suggested at one time or another.

In order to establish the origin of EHes and related objects, temperatures and gravities are required. Additional observables are provided by the surface composition and by the fact that many EHes are pulsating. Pulsations provide an opportunity to make direct measurements of mass and radius. The talk of which this report is a summary reviewed the spectroscopic data on surface composition, summarized the principal theories for the evolutionary origin, and discussed the major pulsation properties of EHes. There is only space within these proceedings to present the latter, together with a synopsis of the extraordinary pulsating EHe V652 Herculis. The complete version of this paper is published online (Jeffery 2013). It includes a summary of the spectroscopic data, and a more detailed review of arguments which currently favour the origin of EHes as the product of a double white dwarf merger.

\section{Pulsation}

\subsection{Discovery and classification}

Landolt (1975) made the first detection of variability in an EHe, the hot star HD $160641=$ V2076 Oph which showed a brightening by 0.1 mag over seven hours. The discovery of a 


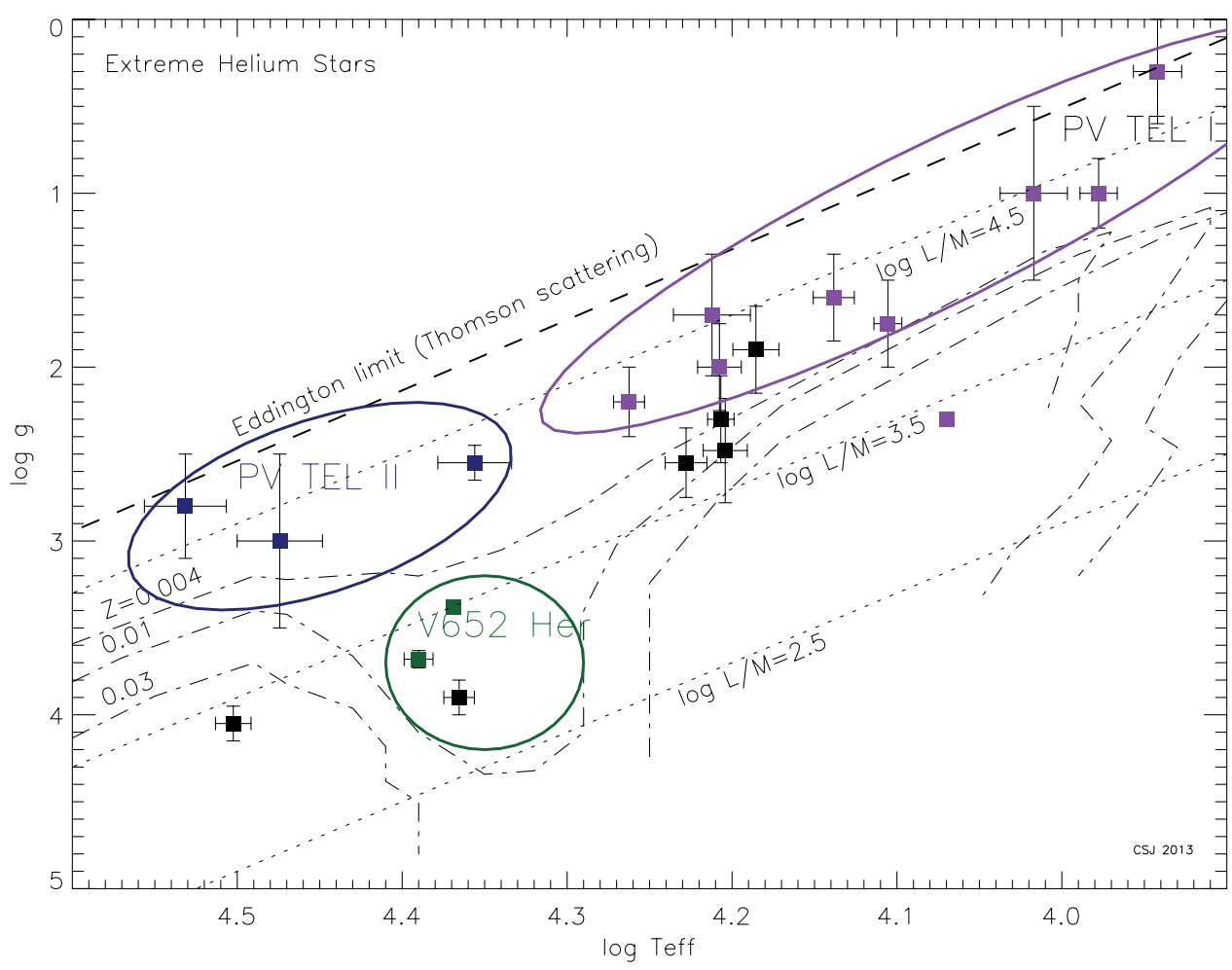

Figure 2. The $\log g-\log T_{\text {eff }}$ diagram for EHe variables, adapted from Jeffery (2008c), including the position of the Eddington limit (assuming Thomson scattering: dashed) and the loci of stars with given luminosity-to-mass ratios (solar units: dotted). Stars above the boundaries shown for metallicities $Z=0.004,0.01,0.03$ (dot-dash) are predicted to be unstable to pulsations (Jeffery \& Saio 1999). Ellipses (coloured in electronic version) identify three groups of pulsating helium stars. In the electronic version, PV TEL I variables are shown in purple, PV TEL II variables in blue, and V652 Her variables in green. Non-variables are black.

0.108 d pulsation period in V652 Her (Landolt 1975) prompted searches for short-period variability in other EHes. Walker \& Hill (1985) reported irregular small-amplitude variations of weeks to months in HD $168476=\mathrm{PV}$ Tel, thus codifying the PV Tel variables as a class. Bartolini et al. (1982) reported a short-period variation in $\mathrm{BD}+10^{\circ} 2179=\mathrm{DN}$ Leo which could not be verified (Hill et al. 1984; Grauer et al. 1984). From 1983 - 1988, a St Andrews/SAAO campaign made discoveries of variability in six EHe stars, namely FQ Aqr, NO Ser, V2205 Oph, V2244 Oph and V1920 Cyg (Jeffery \& Malaney 1985; Jeffery et al. 1986, 1985; Morrison 1987; Morrison \& Willingale 1987). As with DN Leo, variability was not confirmed in Popper's star HD $124448=$ V821 Cen (Jeffery \& LynasGray 1990). In the following decade, variability was discovered in V4732 Sgr, V5541 Sgr and V354 Nor (Lawson et al. 1993; Lawson \& Kilkenny 1998), and most recently in the enigmatic MVSgr (Percy \& Fu 2012). Following a prediction by Saio (1995), Kilkenny \& Koen (1995) discovered $0.1 \mathrm{~d}$ pulsations in BX Cir. The distribution of variable and non-variable EHes is shown in Fig. 2. Jeffery (2008c) gives a complete list of properties including original catalogue numbers, GCVS variable star designations, approximate periods, temperatures, and gravities.

The first discovery of the St Andrews/SAAO campaign concerned FQ Aqr, which showed a $21.2 \mathrm{~d}$ sinusoidal oscillation with an amplitude of 0.4 mag in $V$ and 0.05 mag in $b-y$. Jeffery \& Malaney (1985) associated this with pulsation. Together with periods for 
other EHes, a pulsation-period effective-temperature relation of the form $\Pi \propto T_{\text {eff }}^{3}$ was apparent. For stars of the same luminosity, this corresponds to the period mean-density relation for classical radial pulsators. On the basis of this inference, Saio \& Jeffery (1988) calculated a series of linear non-adiabatic pulsation models for low-mass high-luminosity stars in the temperature range 7000 to $30000 \mathrm{~K}$. They showed that, for sufficiently high values of $L / M$, opacity-driven strange-mode radial pulsations would be excited, consistent with the periods observed in the cooler PV Tel variables (Fig. 2). Jeffery (2008c) calls these "Type I" PV Tel variables.

At $T_{\text {eff }}>20000 \mathrm{~K}$, both V2205 Oph and V2076 Oph were apparently multiperiodic on timescales longer than that consistent with a fundamental radial pulsation, and had been inferred to be g-mode non-radial pulsators (Jeffery et al. 1985; Lynas-Gray et al. 1987). Together with V5541 Sgr, Jeffery (2008c) calls these "Type II" PV Tel variables.

The short-period variability in the lower luminosity star V652 Her was not explained until the introduction of OPAL opacities (Rogers \& Iglesias 1992), when Saio (1993) showed that, in the absence of hydrogen, The $Z$-bump opacity mechanism could easily drive radial pulsations with normal (solar-like) metallicities. Jeffery (2008c) puts BX Cir and V652 Her in the same class, but they would be better labelled V652 Her variables (Fig. 2).

\subsection{Pulsation properties}

What promised to be a class of simply periodic variables with a strict period-temperature relation and the possibility of measuring direct radii using Baade's method turned out to be a chimæra. A second season of observations of FQ Aqr did not reveal a unique period, with $21.5 \mathrm{~d}$ and $23.0 \mathrm{~d}$ being possible (Jeffery et al. 1986). Kilkenny et al. (1999) monitored both FQ Aqr and NO Ser for five consecutive seasons and, from the Fourier power spectrum, reported the "apparent presence of several periods but, if real, none seems to persist for more than one season." Data from SuperWASP (unpublished), and a wavelet analysis of the Kilkenny et al. data are similarly ambiguous. The only recurring signal occurs at a period of around $20 \mathrm{~d}$, but is not coherent over a long period of time.

The absence of a regular period is not fatal for extracting stellar radii, so long as the oscillation is radial and angular radii and radial velocities can be measured simultaneously. Radial-velocity amplitudes of a few $\mathrm{km} \mathrm{s}^{-1}$ were measured in PV Tel, FQ Aqr and V2244 Oph at the same time as angular radius measurements were obtained with IUE (Jeffery et al. 2001a). Relative phases were consistent with a radial pulsation. In two cases, the resulting radius and mass measurements were consistent with theoretical expectation, but the relative mass errors did not provide a strong constraint for establishing an evolutionary origin.

The aperiodicity of FQ Aqr is reflected in observations of other EHe stars (cf. Walker \& Hill 1985). Wright et al. (2006) considerably extended the photometry and spectroscopy of V2076 Oph. Instead of recovering the periods of 0.7 and $1.1 \mathrm{~d}$ reported by Lynas-Gray et al. (1987), they reported that "conventional Fourier analysis ... fails to reveal coherent frequencies" and suggest that the light curve "could be a result of random variations". On the other hand, the high-resolution spectrum of V2076 Oph shows prominent line-profile variability (LPV) on a timescale of a few hours which is symptomatic of a non-radial pulsation. Of note is that, although LPVs are visible in all He I lines, they are only seen in the He II $4686 \AA$ line, and not in other He II lines (Fig. 2: Jeffery 2008b). Such intriguing results are almost impossible to interpret in isolation from photometric monitoring and over such a short time interval. 


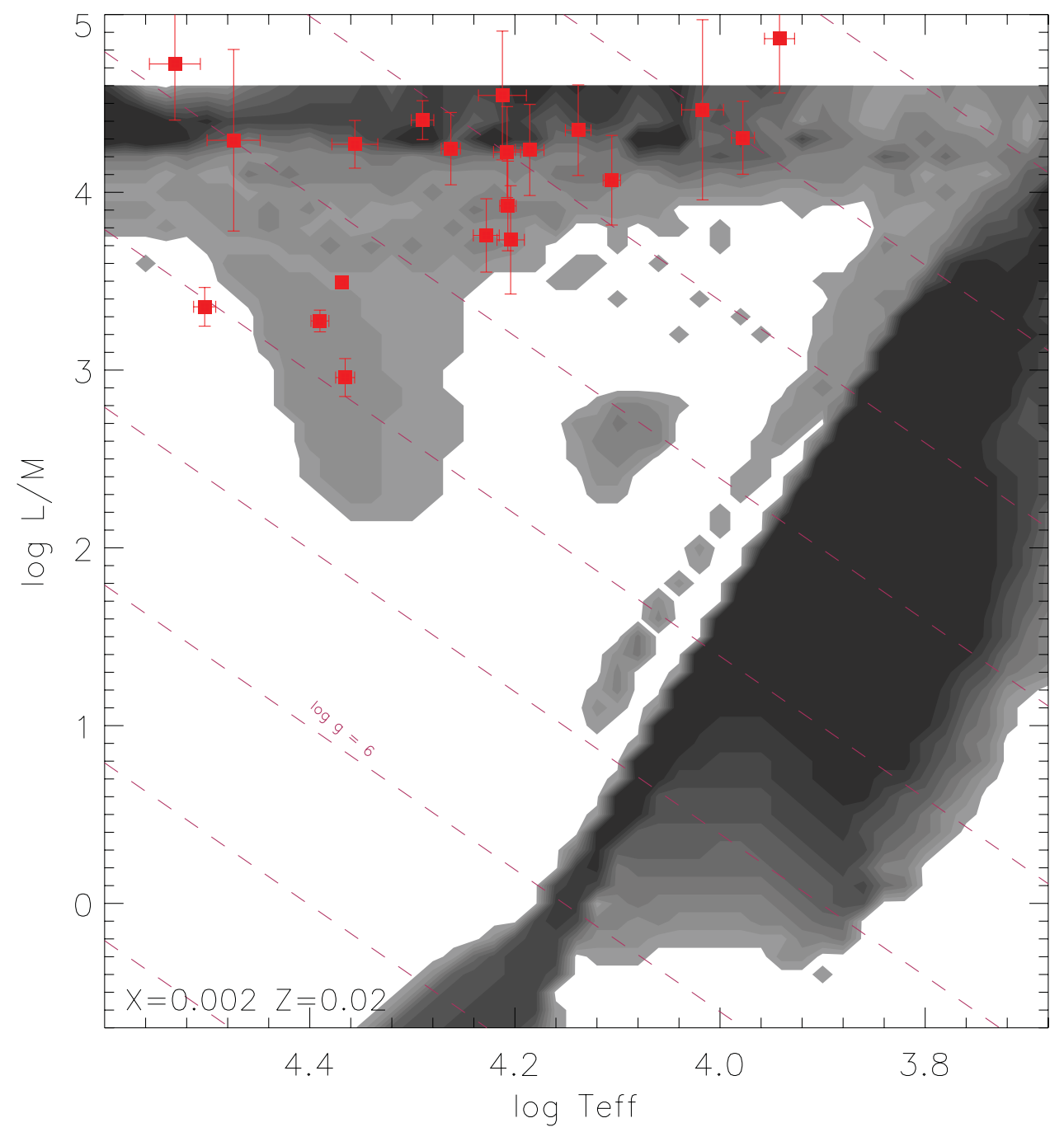

Figure 3. Contour plot showing the number of unstable radial modes with $n<15$ in linear non-adiabatic pulsation analyses of hydrogen-deficient stellar envelopes. The ordinate is $\log L / M$ in solar units, and the abscissa is $\log T_{\text {eff }}$. The plot is virtually invariant to the mass, at least in the range $0.2-1.0 M_{\odot}$. The solid squares correspond to EHe stars. White means no unstable modes except for $\log L / M>4.6$ where model envelopes are difficult to compute. Surface gravity contours for $\log g=8,7,6, \ldots$ are represented as broken lines.

\subsection{Theoretical considerations}

Jeffery \& Saio (2013) extended their investigations of pulsation stability as a function of hydrogen abundance (mass fraction: $X)$ to a larger range of effective temperature $\left(T_{\text {eff }}\right)$, luminosity-to-mass ratio $(L / M)$ and $X$ than before (Jeffery \& Saio 1999). Models for $0.9>X>0.002$ were computed. Figure 2 shows the instability domain for $X=0.002$ and solar metallicity. At large $X$, the classical Cepheid instability strip is clearly seen. At high $L / M$ strange modes are excited. As $X$ is reduced, the $Z$-bump instability finger starts to develop, and a strip of intermediate-order radial modes develops blueward of the classical instability strip. The strange modes are less sensitive to $X$, and at low $X$ correspond to the observed locations of the variable EHe stars (Fig. 3). At low metallicity, 
the $Z$-bump finger is much diminished, and the lower edge of the strange-mode domain moves to higher $L / M$ ratio (Fig. 2), so the absence of variability in metal-poor EHes is anticipated (Saio \& Jeffery 1988; Saio 1993). With no hydrogen, the morphology of the classical strip again changes and other pockets of instability appear, in particular one at $T_{\text {eff }} \approx 13000 \mathrm{~K}$ and $L / M \approx 500 L_{\odot} / M_{\odot}$. It remains to be seen whether any real hydrogen-deficient stars can be identified with such models.

Strange-mode oscillations in stars with high $L / M$ ratios have been identified for some time (Wood 1976). They appear theoretically in non-adiabatic pulsation analyses and have no corresponding modes in the adiabatic approximation. In particular, they appear to be associated with stellar envelopes where high opacities in the ionisation zones also lead to a density inversion - effectively creating a radiation-pressure dominated cavity in the stellar interior. They are discussed at greater length by, inter alia, Gautschy \& Glatzel (1990) and Saio et al. (1998).

Evidence that non-radial oscillations may be present in the hottest EHes led Guzik et al. (2006) to find a large number of unstable opacity-driven g-modes in models for V2076 Oph. It will be interesting to learn whether extreme non-adiabacity and strangemode characteristics or frequent switching between closely-spaced modes is responsible for the absence of long-lived coherent periods in these stars.

\section{The shocking case of V652 Herculis}

$\mathrm{BD}+13^{\circ} 3224=\mathrm{V} 652 \mathrm{Her}$ is an extreme helium star and a B-type giant discovered by Berger \& Greenstein (1963). With a nitrogen-rich, carbon-poor surface, it pulsates with a period of $0.108 \mathrm{~d}$ which is decreasing at a substantial rate (cf. Kilkenny \& LynasGray 1982; Kilkenny et al. 2005). This period decrease implies a radius contraction that, together with a radius $2.31 \pm 0.02 R_{\odot}$ and mass $0.59 \pm 0.18 M_{\odot}$ (Jeffery et al. $2001 \mathrm{~b}$ ), in turn implies that V652 Her is evolving to become a hot subdwarf within $\leqslant 10^{5}$ yr (Jeffery 1984). Single-star models cannot explain the origin of V652 Her, which has an almost completely CNO-processed atmosphere (Jeffery et al. 1999). On the other hand, models for the post-merger evolution of two helium white dwarfs very successfully match nearly all of the observational properties (Saio \& Jeffery 2000), as well as demonstrating that such mergers will become helium-rich hot subdwarfs (Zhang \& Jeffery 2012b).

Saio (1993) demonstrated that $Z$-bump opacity instability drives fundamental-mode radial pulsations in V652 Her. The radial-velocity curve shows that, for nine tenths of the cycle, the surface layers are nearly ballistic (Hill et al. 1981; Lynas-Gray et al. 1984; Jeffery \& Hill 1986). Close to minimum radius, the surface acceleration is so large that the atmosphere may be shocked (Jeffery et al. 2001b). Since hydrogen-deficient atmospheres are in general more transparent than hydrogen-rich ones, V652 Her provides a unique opportunity to study the dynamical behaviour of a pulsating atmosphere at greater depths (or densities) than is usually the case. Suitable observations could test non-linear hydrodynamic models for pulsation (Fadeyev \& Lynas-Gray 1996; Montañés Rodríguez \& Jeffery 2002). Jeffery et al. (2013a) present a more extended account.

High-speed spectroscopy of V652 Her was obtained with the Subaru High Dispersion Spectrograph. 2 nights of observations cover over six pulsation cycles with a temporal resolution of 174 seconds. These observations aim to identify structure in the line cores around minimum radius, to resolve the passage of the wave through the photosphere using ions with different ionisation potentials, to determine whether this generates a shock front, or whether the photosphere adjusts subsonically, and to establish how close the "free-fall" phase is to a true ballistic trajectory. The interpretation of the observations requires coupling a hydrodynamic model of the pulsation to a radiative transfer code, 
so that the emergent spectrum can be computed realistically. A summary of progress is given by Jeffery et al. (2013a,b).

\section{Conclusion}

Extreme helium stars form a group of some 15 low-mass supergiants of spectral types A and B. Their helium-dominated atmospheres are extremely hydrogen poor $(<0.1 \%)$ and carbon-rich $(1-3 \%)$. Evidence from kinematics, surface composition and distribution in effective temperature and surface gravity points to a strong link with the cooler R CrB stars, and to their origin in the merger of a helium white dwarf with a carbon-oxygen white dwarf.

Most EHe stars are photometric variables with amplitudes around one tenth of a magnitude. Most are also radial-velocity variables with amplitudes of a few $\mathrm{km} \mathrm{s}^{-1}$. The timescales of these variations as a function of effective temperature are consistent with the stellar dynamical timescales and hence with being due to pulsations. Theoretical models show most EHes to be unstable to opacity-driven radial pulsations. However, in all but two cases (V652 Her and BX Cir), the observed variations are not strictly periodic. Additional work is necessary to better characterise the periods and amplitudes and to obtain radii using the Baade-Wesselink method.

V652 Her and BX Cir are less luminous than the majority of EHes. V652 Her can be explained by the merger of two helium white dwarfs evolving to become a helium-rich subdwarf. The carbon-rich BX Cir is harder to explain, but could be similar. Both stars pulsate radially with periods of $0.1 \mathrm{~d}$. The radial-velocity curve of V652 Her is nearly ballistic, with a very steep acceleration phase. New observations and models provide a unique dataset and toolkit for exploring the physics of these pulsations.

\section{Bibliography}

Bartolini, C., Bonifazi, A., Fusi Pecci, F., et al. 1982, Ap\&SS, 83, 287

Berger, J. \& Greenstein, J. L. 1963, PASP, 75, 336

Fadeyev, Y. A. \& Lynas-Gray, A. E. 1996, MNRAS, 280, 427

Gautschy, A. \& Glatzel, W. 1990, MNRAS, 245, 597

Grauer, A. D., Drilling, J. S., \& Schönberner, D. 1984, A\&A, 133, 285

Guzik, J. A., Peterson, B. R., Cox, A. N., \& Bradley, P. A. 2006, MemSAIt, 77, 131

Hill, P. W., Kilkenny, D., Schönberner, D., \& Walker, H. J. 1981, MNRAS, 197, 81

Hill, P. W., Lynas-Gray, A. E., \& Kilkenny, D. 1984, MNRAS, 207, 823

Jeffery, C. S. 1984, MNRAS, 210, 731

Jeffery, C. S. 2008a, $A S P-C S, 391,3$

Jeffery, C. S. 2008b, $A S P-C S, 391,53$

Jeffery, C. S. 2008c, IBVS, 5817

Jeffery, C. S. 2013, arXiv: 1311.1635

Jeffery, C. S. \& Hill, P. W. 1986, MNRAS, 221, 975

Jeffery, C. S. \& Lynas-Gray, A. E. 1990, MNRAS, 242, 6

Jeffery, C. S., Malaney, R. A. 1985, MNRAS, 213, 61P

Jeffery, C. S. \& Saio, H. 1999, MNRAS, 308, 221

Jeffery, C. S. \& Saio, H. 2013, MNRAS, 435, 885

Jeffery, C. S., Skillen, I., Hill, P. W., Kilkenny, D., Malaney, R. A., \& Morrison, K. 1985, MNRAS, 217, 701

Jeffery, C. S., Drilling, J. S., \& Heber, U. 1987, MNRAS, 226, 317

Jeffery, C. S., Hill, P. W., \& Morrison, K. 1986, in: K. Hunger, D. Schönberner, N. Kameswara Rao (eds.), Hydrogen-Deficient Stars and Related Objects, Proc. IAU Colloqium No. 87, Astrophysics and Space Sci. Library, Vol. 128 (Dordrecht: D. Reidel Publishing Co.) p. 95 
Jeffery, C. S., Hill, P. W., \& Heber, U. 1999, A\&SA, 346, 491

Jeffery, C. S., Starling, R. L. C., Hill, P. W., \& Pollacco, D. 2001a, MNRAS, 321, 111

Jeffery, C. S., Woolf, V. M., \& Pollacco, D. L. 2001b, A\&SA, 376, 497

Jeffery, C. S., Shibahashi, H., Kurtz, D., Elkin, V., Montañés-Rodríguez, P., \& Saio, H. 2013a, in: H. Shibahashi, A. E. Lynas-Gray (eds.), Progress in Physics of the Sun and Stars: A New Era in Helio- and Asteroseismology, ASP-CS, in press

Jeffery, C. S., Shibahashi, H., Kurtz, D., Elkin, V., Montañés- Rodríguez, P., \& Saio, H. 2013b, in: V. Van Grootel, E. Green, G. Fontaine, S. Charpinet (eds.), Hot Subdwarf Stars and Related Objects, $A S P-C S$, in press

Kilkenny, D. \& Koen, C. 1995, MNRAS, 275, 327

Kilkenny, D. \& Lynas-Gray, A. E. 1982, MNRAS, 198, 873

Kilkenny, D., Lawson, W. A., Marang, F., Roberts, G., \& van Wyk, F., 1999, MNRAS, 305, 103

Kilkenny, D., Crause, L. A., \& van Wyk, F. 2005, MNRAS, 361, 559

Landolt, A. U. 1975, ApJ, 196, 789

Lawson, W. A. \& Kilkenny, D. 1998, Observatory, 118, 1

Lawson, W. A., Kilkenny, D., van Wyk, F., Marang, F., Pollard, K., \& Ryder, S. D. 1993, MNRAS, 265, 351

Lynas-Gray, A. E., Schönberner, D., Hill, P. W., \& Heber, U. 1984, MNRAS, 209, 387

Lynas-Gray, A. E., Kilkenny, D., Skillen, I., \& Jeffery, C. S. 1987, MNRAS, 227, 1073

Montañés Rodríguez, P. \& Jeffery, C. S. 2002, MNRAS, 384, 433

Morrison, K. 1987, MNRAS, 224, 1083

Morrison, K. \& Willingale, G. P. H. 1987, MNRAS, 228, 819

Percy, J. R. \& Fu, R. 2012, Journal AAVSO, 40, 900

Pigott, E. \& Englefield, H. C. 1797, Phil. Trans. Ser. I, 87, 133

Popper, D. M. 1942, PASP, 54, 160

Rogers, F. J. \& Iglesias, C. A. 1992, ApJS, 79, 507

Saio, H. 1993, MNRAS, 260, 465

Saio, H. 1995, MNRAS, 277, 1393

Saio, H. \& Jeffery, C. S. 1988, ApJ, 328, 714

Saio, H. \& Jeffery, C. S. 2000, MNRAS, 313, 671

Saio, H., Baker, N. H., \& Gautschy, A. 1998, MNRAS, 294, 622

Schönberner, D. 1975, A\& A, 44, 383

Walker, H. J. \& Hill, P. W. 1985, A\& $A S, 61,303$

Wood, P. R. 1976, MNRAS, 174, 531

Wright, D. J., Lynas-Gray, A. E., Kilkenny, D., et al. 2006, MNRAS, 369, 2049

Zhang, X. \& Jeffery, C. S. 2012, MNRAS, 419, 452 